\title{
The Influence of the Two-child Policy on China's Population Projection
}

\author{
Hao $\mathrm{Yan}^{1}$ \\ ${ }^{1}$ Software Engineering, Information Science and Technology College, Dalian Maritime University, China \\ Correspondence: Hao Yan, Software Engineering, Information Science and Technology College, Dalian Maritime \\ University, Dalian, Liaoning, China.
}

Received: March 15, 2017 Accepted: March 30, 2018 Online Published: April 18, 2018

doi:10.5539/ijsp.v7n3p94

URL: https://doi.org/10.5539/ijsp.v7n3p94

\begin{abstract}
Since January 1,2016, China has implemented the Two-child policy. The Two-child policy is contrast to the One-child policy which has been implemented for nearly 30 years by Chinese government. Since this Two-child policy is a basic national policy of China, its purpose is to determine a reasonable rate of population development. Whether the two-child policy can really achieve the desired effect. This article will discuss statistical methods and social realities.

Firstly, author uses the entropy weight with Analytic Hierarchy Process(AHP) method. Eighteen indicators affecting the population were set up to establish an initial evaluation matrix according to the actual situation and determine the accurate weight of each indicator.

Secondly, the author makes predictions on China's future population. In order to make sure the accuracy of the forecast, author makes second forecast of the modified value to reduce the noise caused by the entropy weight method. And the author discusses which method is suit for this question.
\end{abstract}

Finally, author analyzes this situation.

Keywords: Two-child policy, entropy weight method, Analytic Hierarchy Process (AHP), the iterative prediction method, grey prediction, BP neural network

\section{Introduction}

In October 2015, the communique of the Fifth Plenary Session of the Eighteenth Central Committee of the Communist Party of China pointed out: author must adhere to the basic national policy of family planning, actively implement the action of responding to population aging, and implement a comprehensive two-child policy. From January 1, 2016, China has implemented the two-child policy.

The two-child policy is a kind of family planning policy implemented by China. Many discussions in the community have focused on the following aspects: whether the relationship between the comprehensive two-child policy and fertility rates will lead to childbearing peaks; whether it will slow down the pace of social aging, or even reverse the situation; whether it can increase the labor force; whether it can ease the balance of supply and demand of the labor force after several years and prevent China from falling into the middle income trap.

The authors proceeding this study of the "two-child policy" on the Chinese population will be conducted to provide quantitative basis for relevant policies and scientific decisions, and to try to solve the problem of future Chinese population prediction. Compared with traditional methods, this paper adopts more influencing factors, and the prediction methods studied in this paper are used to make comparative judgments, which can further improve the accuracy of prediction.

First, select a suitable evaluation index system to quantify the impact of the comprehensive two-child policy on the Chinese population and establish a mathematical model for the Chinese population. The population model established was used to make predictions and quantitatively compare the impact on the population problem in China during and after the implementation of the two-child policy. Finally, based on the results of the study, from the perspective of government decision-making, the rationalization of the comprehensive implementation of the two-child policy is proposed to make the development of the population more in line with the progress of society, increase the fertility rate, reduce the aging of the society, increase the labor force, and promote others. The simultaneous development of health care. 


\section{Assumption and Data}

\subsection{Model Assumption}

1.Assume that our investigation during the research phase is not affected by natural factors such as disease and war;

2.Assume that the growth level of the Chinese population is affected by the indicators author sets;

3.The indicators author chooses can accurately reflect the population growth level;

4.Assume that the data on the demographic data obtained are correct and correct. The future national economy will develop steadily and the population policy will remain unchanged.

5.Assume that the Chinese people's concept of fertility and fertility are basically unchanged;

\subsection{Variables in the Study}

Table 1. Description of elements in this study

\begin{tabular}{c|l}
\hline$x_{i j}$ & Initial evaluation matrix \\
\hline$\rho_{i j}$ & The weighting method calculates the proportion \\
\hline$e_{j}$ & The j-th index of entropy \\
\hline$d_{j}$ & Information Entropy Redundancy \\
\hline$\omega_{j}$ & The weight of each indicator \\
\hline$C$ & Decision matrix \\
\hline$c_{j}^{*}$ & Positive ideal solution \\
\hline$c_{j}^{0}$ & Negative ideal solution \\
\hline$E_{j}$ & Positive ideal solution \\
\hline$\lambda_{(k)}$ & Sequence ratio \\
\hline$y^{(0)}(k)$ & The translation data of the original data is updated \\
\hline$b$ & Gray effect \\
\hline$\mu$ & Parameter vector \\
\hline$a$ & Development coefficient \\
\hline$z_{j}$ & Comprehensive weight \\
\hline$\varepsilon^{(0)}$ & Residual coefficient \\
\hline
\end{tabular}

\subsection{Indicators}

Table 2. Comprehensive evaluation indicators system

\begin{tabular}{|c|c|c|}
\hline First-level indicators & Second-level indicators & Third-level indicators \\
\hline \multirow{10}{*}{ Population development indicator system } & \multirow[t]{3}{*}{ Population quality } & Public health expenditure per unit of GDP \\
\hline & & Education expenditure per unit of GDP \\
\hline & & Average years of schooling \\
\hline & \multirow[t]{4}{*}{ Population distribution } & Birth rate \\
\hline & & Neonatal mortality rate \\
\hline & & Natural population growth rate \\
\hline & & In line with policy fertility rate \\
\hline & \multirow[t]{3}{*}{ Population structure } & Child dependency ratio \\
\hline & & Elderly dependency ratio \\
\hline & & Gender structure \\
\hline \multirow[b]{7}{*}{ Economic and social indicator system } & \multirow[t]{4}{*}{ Population and economy } & Per capita annual income \\
\hline & & Tertiary industry employment \\
\hline & & Rural per capita housing area \\
\hline & & Urban per capita housing area \\
\hline & \multirow[t]{3}{*}{ Population and society } & Internet penetration rate \\
\hline & & Number of participating in endowment insurance \\
\hline & & Number penetration rate \\
\hline Environmental indicator & Population and resources & Per capita water resources $\left(\mathrm{m}^{3} /\right.$ person $)$ \\
\hline
\end{tabular}


Collecting sufficient data is the basis of developing a complete indicators system. Author searched the database and found indicators of different population indicators. The data is from the National Bureau of Statistics of the People's Republic of China.

\section{Model and Analysis}

\subsection{The Entropy Weight Method With AHP}

\subsubsection{The Initialization of Matrix}

Firstly, author has to choose 2 countries and 18 indexes, then author needs to establish $\mathrm{x}_{i j}$ which is the $\mathrm{j}$-th index of the i-th country's number $(i=1, \ldots, m, j=1, \ldots, n)$. By using these numbers, author can make the initialization of matrix .

$$
\left(\mathrm{x}=\left(\mathrm{x}_{i j}\right)_{m * n} \quad(i=1, \ldots, m, j=1, \ldots, n) \quad\right)
$$

\subsubsection{Vector Normalization of Indicator Data}

It is difficult to use these data directly because these data have different unit. In order to make out the composite index , author needs to make standardization so that all data will have a common unit . author can change the absolute value of the indicator into a relative value to achieve this purpose. What's more, author needs to use different methods to make data processing because positive and negative indicators have different meanings. (The higher the positive index value the better, the lower the negative index value the better).

$$
x_{i j}{ }^{\prime}=\left\{\begin{array}{c}
\frac{x_{i j}-\min \left\{x_{1 j}, \ldots x_{m j}\right\}}{\max \left\{x_{1 j}, \ldots x_{m j}\right\}-\min \left\{x_{1 j} \ldots x_{m j}\right\}} \\
\frac{\max \left\{x_{1 j} \ldots x_{m j}\right\}-x_{i j}}{\max \left\{x_{1 j}, \ldots x_{m j}\right\}-\min \left\{x_{1 j}, \ldots x_{m j}\right\}}
\end{array}\right.
$$

$\mathrm{X}_{\mathrm{i}}$ is the positive index, $\mathrm{X}_{\mathrm{j}}$ is the positive index

$x_{i j}{ }^{\prime}$ is the $\mathrm{j}$-th index of the $\mathrm{i}$-th country's number $(\mathrm{i}=1, \ldots, \mathrm{m}, \mathrm{j}=1, \ldots, \mathrm{n})$, then author can get the initialization of matrix $X_{i j}{ }^{\prime}$, in order to be easier to understand, author still uses $x_{i j}{ }^{\prime}$ to mark it.

Its most important feature is that, after normalization, each solution has the same attribute value with a sum of squares of 1 , so it is often used to calculate the Euclidean distance between each solution and some kind of virtual solution (such as ideal solution point or negative ideal solution point).

Before evaluating, the data needs to be dimensioned before the metrics are empowered.

Then the indicator can be empowered.

$$
p_{i j}=\frac{x_{i j}}{\sum_{i=1}^{m} x_{i j}}, \quad i=1, \ldots, m, j=1, \ldots, n
$$

\subsubsection{Objective Indicators of the Weight}

Using Entropy Weight Method to Determine Weight of Index.

In order to get a more objective weight, in this study, the objective weight of the index is calculated by using the entropy method.

The principle of the entropy method is as follows:

Calculate the entropy of the first index

$$
e_{j}=-\frac{1}{\ln m} \sum_{i=1}^{m} p_{i j} \ln p_{i j}, \quad i=1, \ldots, m, j=1, \ldots, n
$$

calculate the entropy redundancy

$$
d_{j}=1-e_{j}, j=1, \ldots, n
$$

Calculate the weight of each indicator

$$
\omega_{j}=\frac{d_{j}}{\sum_{j=1}^{n} d_{j}}, j=1, \ldots, n
$$




\subsubsection{Construct a Normalized Weighted Matrix}

The indicators and weights which are dimensionless can be calculated according to equation to get the weighted matrix.

$$
C=\left(c_{i j}\right)_{m \times n}=\omega_{j} \cdot x_{i j}, \quad i=1, \ldots, m, j=1, \ldots, n
$$

3.1.5 Determine the Positive Ideal $C^{*}$ and Negative Ideal $C^{0}$

When author using TOPSIS (Technique for Order Preference by Similarity to an Ideal Solution) to determine the positive and negative solution, author regards the element of positive solution as the optimal value in the indicator, the element of negative ideal solution can be regarded as the worst value. Due to the relatively complex numerical values, it is cumbersome to calculate the Euclidean distance between each evaluation object and the positive or negative solutions. In order to improve the method, the range of the normalized matrix is $[0,1]$.

Therefore, assuming that the $\mathrm{j}$-th property value of the positive solution $C^{*}$ is $c_{j}^{*}$ and the $\mathrm{j}$-th property value of the negative solution $C^{0}$ is $c_{j}^{0}$.

$$
\begin{aligned}
& \text { The Positive Solution } \mathrm{C}_{j}^{*}=\left\{\begin{array}{l}
\omega_{j,} j \text { is the positive indicator } \\
0_{,} j \text { is the negative indicator }
\end{array} \quad \mathrm{j}=1,2, \ldots, \mathrm{n}\right. \\
& \text { The Negative Solution } \mathrm{C}_{j}^{0}= \begin{cases}0, j \text { is the positive indicator } & \mathrm{j}=1,2, \ldots, \mathrm{n} \\
-\omega_{j,} j \text { is the negative indicator }\end{cases}
\end{aligned}
$$

\subsubsection{Calculate the Euclidean Distance}

According to Euclidean distance, calculate the distance $d_{i}^{*}, d_{i}^{0}$ from each country $i$ to positive and negative ideal point.

$$
\begin{aligned}
& d_{i}^{*}=\left[\sum_{j=1}^{n}\left(c_{i j}-\max _{i}\left\{c_{i j}\right\}\right)^{2}\right]^{1 / 2}, \quad i=1, \ldots, m \\
& d_{i}^{0}=\left[\sum_{j=1}^{n}\left(c_{i j}-\min _{i}\left\{c_{i j}\right\}\right)^{2}\right]^{1 / 2}, \quad i=1, \ldots, m
\end{aligned}
$$

Calculate the distance $d_{i}^{*}$ of each country from the positive ideal solution and the distance $d_{i}^{0}$ of the negative ideal solution using (9) and (10), then calculate the comprehensive evaluation index value $E_{i}$ for each country $i$

$$
E_{i}=\frac{d_{i}^{\mathrm{o}}}{d_{i}^{\mathrm{O}}+d_{i}^{*}} \times 100 \%, \quad i=1, \ldots, m
$$

$E_{i}$ reflects the close relationship between the target solution and the ideal solution. When the object of evaluation is closer to positive ideal solution, the closer $E_{i}$ is to $100 \%$, the better the evaluation object level.

\subsubsection{Model Results and Analysis}

Table 3. Distance and comprehensive value

\begin{tabular}{l|l|l|l}
\hline & Positive distance & Negative distance & Comprehensive evaluation value \\
\hline China & 0.1287 & 0.1287 & 0.5366 \\
\hline India & 0.1087 & 0.1087 & 0.5171 \\
\hline
\end{tabular}

Although the entropy weight method can calculate the weight of each indicator more accurately. But this indicator is affected by the individual data. Therefore, the author has combined the Analytic Hierarchy Process to calculate further. 


\subsection{The Entropy Weight Method(EWM) Combine With AHP To Construct Judgment Matrix}

\subsubsection{Construct Judgment Matrix}

Relative Importance Scale $a_{i j}$, i-th compare to j-th:

$$
A=\left(a_{i j}\right)_{n \times n}=\left(\begin{array}{cccc}
a_{11} & a_{12} & \cdots & a_{1 n} \\
a_{21} & a_{22} & \cdots & a_{2 n} \\
\cdots & \cdots & \cdots & \cdots \\
a_{n 1} & a_{n 2} & \cdots & a_{n n}
\end{array}\right)
$$

\subsubsection{Consistency Test}

For the judgment matrix

$$
C I=\frac{\lambda_{\max }-n}{n-1} \quad C R=\frac{C I}{R I}
$$

Finally, the calculation of the maximum eigenvalue is $\lambda_{\max }=21$

$$
C I=\frac{\lambda_{\max }-n}{n-1}=\frac{21-18}{18-1}=0.176 \quad C R=\frac{C I}{R I}=\frac{0.176}{1.70}=0.104 \approx 0.1
$$

Although slightly larger than 0.1 , author still considers the matrix to be consistent.

3.2.3 Combine with AHP

$$
z_{j}=\frac{\omega_{j} s_{j}}{\sum_{j=1}^{n} \omega_{j} s_{j}}, j=1, \ldots, n
$$

Table 4. The new weight

\begin{tabular}{l|c}
\hline \multicolumn{1}{c|}{ indicators } & new weight \\
\hline In line with the policy of fertility & 0.124369448 \\
\hline Natural population growth rate & 0.056480331 \\
\hline Birth rate & 0.047068366 \\
\hline Neonatal mortality & 0.068287632 \\
\hline Public health expenditure per unit of GDP & 0.005014728 \\
\hline Education expenditure per unit of GDP & 0.000738466 \\
\hline Average years of schooling & 0.045118363 \\
\hline Child dependency ratio & 0.045974538 \\
\hline Elderly dependency ratio & 0.014922889 \\
\hline Gender structure & $5.13 \mathrm{E}-05$ \\
\hline Per capita annual income (US dollars) & 0.206740006 \\
\hline Tertiary industry employment (ten thousand people) & 0.013873385 \\
\hline Rural per capita housing area & 0.02785009 \\
\hline Urban per capita housing area & 0.022536127 \\
\hline Number of people participating in pension insurance & 0.152497976 \\
\hline Number of people participating in unemployment insurance & 0.161833164 \\
\hline Internet penetration rate & 0.005382035 \\
\hline Per capita water resources & 0.001261155 \\
\hline Weights are obtained, the next Step an be predicted
\end{tabular}

After the new weights are obtained, the next step can be predicted. 


\section{The Choice and Estimating of Prediction Method}

\subsection{Establish a GM(1,1) Model}

The grey model is been constructed. Let $x^{(0)}$ be the nonnegative original data sequence, $x^{(1)}$ is the first-order cumulative summation sequence of $x^{(0)}, z^{(1)}$ is the generating sequence of equalizations for $x^{(1)}$ 。

$$
\begin{aligned}
& x^{(0)}=\left\{x^{(0)}(1), x^{(0)}(2), \ldots, x^{(0)}(n)\right\} \\
& x^{(1)}=\left\{x^{(1)}(1), x^{(1)}(2), \ldots, x^{(1)}(n)\right\}
\end{aligned}
$$

In those two lines $\quad x^{(1)}(k)=\sum_{j=1}^{k} x^{(0)}(j), k=1, \ldots, n$

$$
z^{(1)}=\left\{z^{(1)}(2), z^{(1)}(3), \ldots, z^{(1)}(n)\right\}
$$

In this line $z^{(1)}(k)=\left(x^{(1)}(k)+x^{(1)}(k-1)\right) / 2, k=2,3, \ldots, n$

Establish the model of gray differential equation

The sequence $x^{(1)}(k)$ has an approximate law of exponential growth, and the solution to the first-order equation is exactly the exponential form, hence it can be considered that the new sequence satisfies the first-order linear differential equation. The gray differential equation model of GM $(1,1)$ can be defined as:

$$
\frac{d x^{(1)}(k)}{d t}+a z^{(1)}(k)=b
$$

So

$$
x^{(0)}(k)+a z^{(1)}(k)=b
$$

In this two equations, is development factor which reflects the development trend of $x^{(1)}$ and the original sequence; $b$ is the amount of gray, which reflects the transformation relationship between the data; $z^{(\mathrm{l})}(k)$ is the background value of albino.

Substitute $k=2,3, \ldots, n$ into the later equation

$$
\left\{\begin{array}{c}
x^{(0)}(2)+a z^{(1)}(2)=b \\
x^{(0)}(3)+a z^{(1)}(3)=b \\
\vdots \\
x^{(0)}(n)+a z^{(1)}(n)=b
\end{array}\right.
$$

Correspondingly

$$
\begin{gathered}
Y=\left(x^{(0)}(2), x^{(0)}(3), \ldots, x^{(0)}(n)\right)^{T} \\
B=\left[\begin{array}{cc}
-z^{(1)}(2) & 1 \\
-z^{(1)}(3) & 1 \\
\vdots & \vdots \\
-z^{(1)}(n) & 1
\end{array}\right]=\left[\begin{array}{cc}
-0.5\left[x^{(1)}(1)+x^{(1)}(2)\right] & 1 \\
-0.5\left[x^{(1)}(2)+x^{(1)}(3)\right] & 1 \\
\vdots & \vdots \\
-0.5\left[x^{(1)}(n-1)+x^{(1)}(n)\right] & 1
\end{array}\right]
\end{gathered}
$$

So the GM $(1,1)$ model can be expressed as a matrix equation $Y=B \mu$

Among them $\mathrm{Y}$ is the data vector, $\mathrm{B}$ is the data matrix, $\mu$ is the parameter vector.

The least squares estimate of $\mu=(a, b)^{T}$ is satisfied

$$
\hat{\mu}=(\hat{a}, \hat{b})^{T}=\left(B^{T} B\right)^{-1} B^{T} Y
$$

Substituting $\hat{\mu}$ obtained from (3.5) into (3.4) and solving differential equations, the GM (1,1) prediction model is:

$$
\hat{x}^{(1)}(k+1)=\left(x^{(0)}(1)-\frac{b}{a}\right) e^{-a k}+\frac{b}{a}, \quad k=0,1, \ldots, n-1
$$


Restore value:

$$
\left.\hat{x}^{(0}\left({ }^{\prime} k+1\right)\right)^{\wedge} x^{(1}\left(k+1 \text { 1) } x,{ }^{(1} k\right) \quad k \quad 1,2
$$

\subsection{Grey Prediction Model Result}

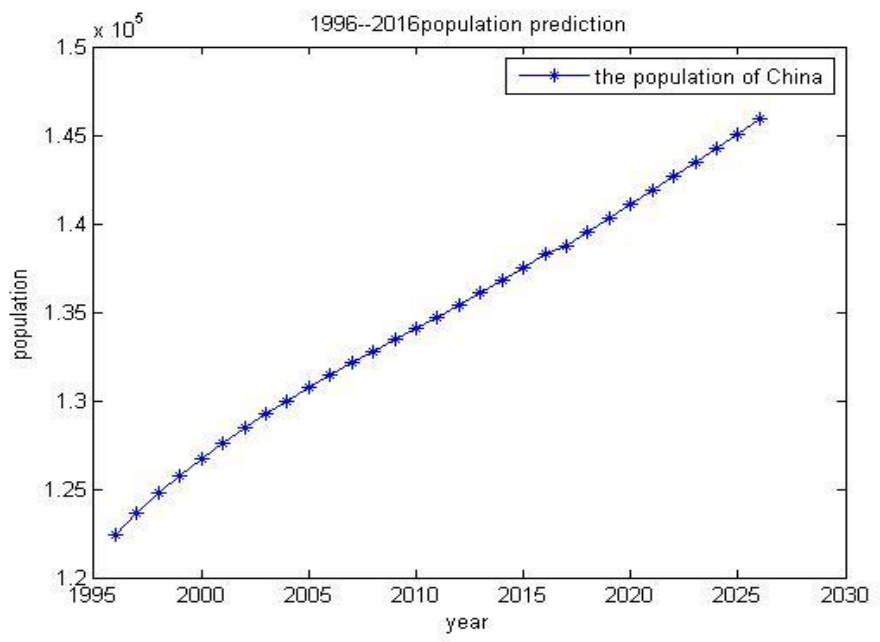

Picture 1.1996 -2016 population prediction

This prediction shows the figure increases smooth as before and Chinese population can't increase significantly as the Chinese government thought. This means that the population ageing cannot be solved. For the grey prediction is applicable to the solution of this problem, a new improved model will be discussed below.

\subsection{Establish Grey-BP Neural Network Model}

Although the grey prediction model can be used to judge the future population, the author still believes that this prediction method still has large deviations and uncertainties, and the original method should be improved. The following author will discuss the use of BP neural network to iteratively improve.

\subsubsection{Determination of BP Network Topology}

The input is population and the output is also population, a simple three-layer BP neural network is designed by experience, with 18 neurons at the input layer and one neuron at the output layer. The hidden layer sets 10 neurons based on empirical formulas to prevent for overfitting, the error learning target of the BP neural network is set to 0.1 , the maximum number of iterations is 3000 , and the learning speed is set to 0.01 .

\section{Selection of transfer function}

Input layer to hidden layer: hyperbolic tangent function $f(x)=\tanh (x)=\frac{1-e^{-2 x}}{1+e^{-2 x}}$, output between $(-1,1)$;

Hidden layer to output layer: linear function $f(x)=x$, the output can be arbitrarily selected.

2. Selection of training functions

The traingdx function is a BP algorithm training function with gradient descent/momentum and adaptive lr. The learning rate is variable and the training performance is better.

\subsubsection{Establish New Forecast Values}

The new residual sequence is predicted by the training model of the BP neural network, which is written as $\hat{\varepsilon}^{(0)}=\left(\hat{\varepsilon}^{(0)}(1), \hat{\varepsilon}^{(0)}(2), \ldots, \hat{\varepsilon}^{(0)}(n)\right)$. Then a new set of prediction values is constructed based on the new residual sequence. For $\hat{y}^{(0)}(k, 1)$, that is $\hat{y}^{(0)}(k, 1)=\hat{x}^{(0)}(k)+\hat{\varepsilon}^{(0)}(1),(k=1, \ldots, n)$.

Since the goodness-of-fit was 0.6829 and the mean squared error was 0.01 , the accuracy of the test sample was higher. The population data residuals from 1996 to 2016 were substituted into the trained neural network system to obtain the predicted values of the corresponding indicators. Compare the predicted value of the indicator with the planned value and adjust accordingly. 


\subsubsection{The Result of Prediction}

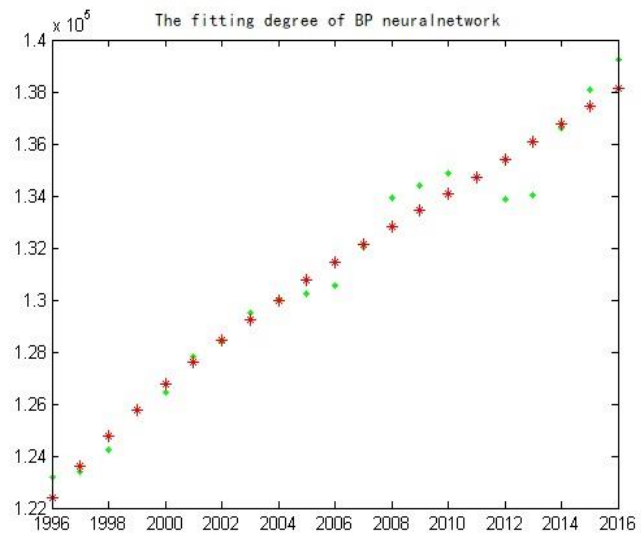

Picture 2. The fitting degree of BP neural network

The red point is real value, and the green one is the prediction value. This shows that it is in line with the situation in the previous decade. Then, author made predictions for the next decade.
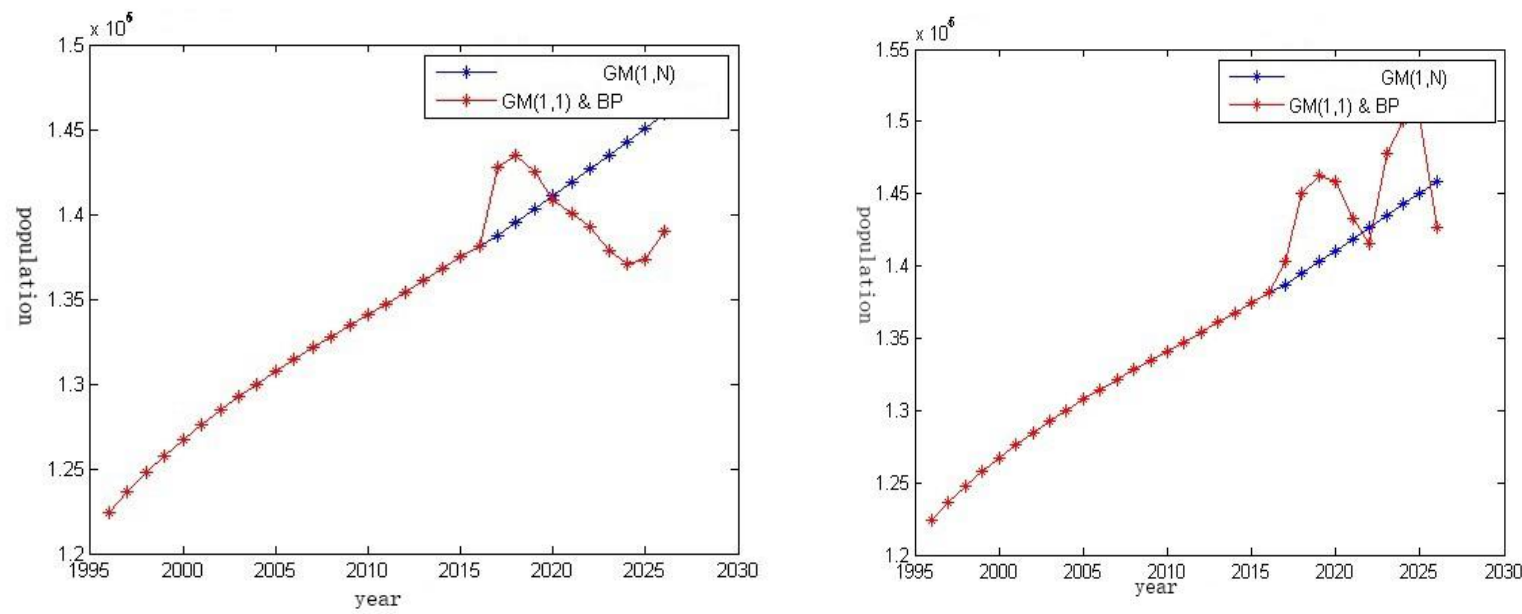

Picture 3. GM(1,N) and GM(1,1)\&BP Neural Network Population Forecasting

Through multiple predictions, the author found that BP neural network is not very stable, so we can make a conclusion that the model is not applicable to the prediction of the Chinese population's prediction. Author believes that the gray prediction is based on a small amount of uncertainty indicators to calculate the most total results, relatively stable, less data, the principle is simple, the operation is convenient, and the short-term prediction accuracy is high. However, in this problem, the BP neural network model cannot determine the exact number of training layers and the number of trainings. The problem does not always give a suitable model condition, and unpredictable errors occur when the number of samples is not enough. Affect the forecast effect. Therefore, when predicting changes in the Chinese population in the short-term future over the next ten years, the grey prediction is more reliable. So, the BP neural network model is abandoned.

Next, the effect of grey prediction will be judged.

\subsubsection{Accuracy Test of Grey Prediction}

To verify whether the gray model meets the requirements in the future, the author conducted a model test. The test of grey prediction model generally includes residual test and posterior differential test.

According to the above, $x^{(0)}$ is the original sequence and $\hat{x}^{(0)}$ is the final prediction sequence. 
(1) Residual test

Absolute error sequence $\Delta^{(0)}(k)=\left|x^{(0)}(k)-\hat{x}^{(0)}(k)\right|, \quad k=1,2, \ldots, n$

Relative error sequence $\quad \varepsilon(k)=\frac{\Delta^{(0)}(k)}{x^{(0)}(k)} \times 100 \%, k=1,2, \ldots n$,

Residual sequence $\varepsilon^{(0)}(k)=x^{(0)}(k)-\hat{x}^{(0)}(k)$

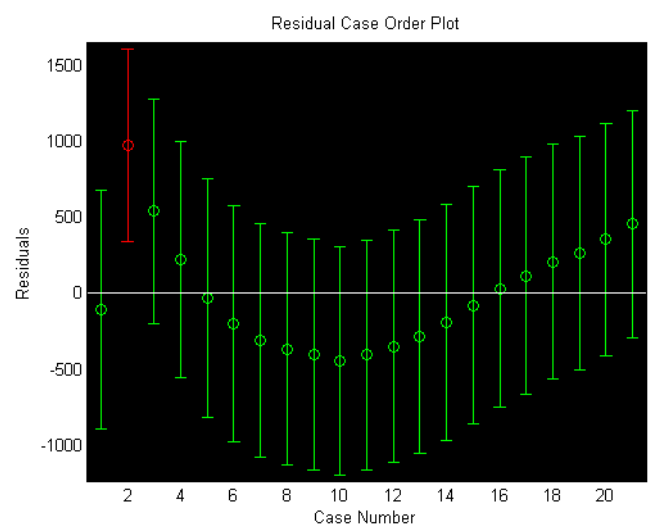

Picture 4. Gray prediction residual map

From the residual graph, author can see that the residual of the data is far from the zero point. When the confidence interval of the residual contains zero point, it means that the regression model can better meet the original data, otherwise it can be regarded as an abnormal point.

Therefore, 1998-2016, is in good agreement with the original data. In 1997, the confidence interval deviated from zero and was an anomaly.

(2) Posterior differential test

$$
\text { Variance ratio } \quad C=\frac{S_{2}}{S_{1}}
$$

$S_{1}$ is the standard deviation of the original sequence

$$
\begin{aligned}
& \bar{x}^{(0)}(k)=\frac{1}{n} \sum_{k=1}^{n} x^{(0)}(k), \quad k=1,2, \ldots, n \\
& S_{1}=\sqrt{\frac{\sum_{k=1}^{n}\left[x^{(0)}(k)-\bar{x}^{(0)}(k)\right]^{2}}{n-1}}, k=1,2, \ldots, n
\end{aligned}
$$

$S_{2}$ is the absolute error sequence standard deviation:

$$
\begin{aligned}
& \bar{\Delta}^{(0)}(k)=\frac{1}{n} \sum_{k=1}^{n} \Delta^{(0)}(k), k=1,2, \ldots, n \\
& S_{2}=\sqrt{\frac{\sum_{k=1}^{n}\left[\Delta^{(0)}(k)-\bar{\Delta}^{(0)}(k)\right]^{2}}{n-1}}, k=1,2, \ldots, n
\end{aligned}
$$

Small error probability $P=\left\{\left|\Delta^{(0)}(k)-\bar{\Delta}^{(0)}(k)\right|<0.6745 S_{1}\right\}$

For a given $C_{0}>0$, when $C<C_{0}$, the model is called the homoscedasticity ratio qualified model; for a given 
$P_{0}>0$, when the time $P>P_{0}$, it is called the small error probability qualified model. Finally, $P=0.905$. The accuracy level is between Level 1 (good) and Level 2 (qualified). author believes that the model predicts better.

Through testing, it can be concluded that the grey prediction has a good effect on the prediction of this problem and can be used as a reliable model.

\subsubsection{The Advantages and Disadvantages of the Model}

Grey system theory has the advantages of less sample data, no need to calculate statistical features, etc. It has been successfully applied in the case of significant uncertainty and lack of data. The BP neural network is very robust and fault-tolerant, because information is distributed in the neurons in the network. Using parallel processing can increase the computational speed correspondingly. However, the neural network method has no definite method for determining the number of hidden layer neurons. There is no unified and complete theoretical guidance for the choice of network structure. Generally, it can only be selected by experience. Due to the contradiction between learning efficiency and stability, it may cause BP neural network to have severe prediction uncertainty.

\section{Analyze}

Why did the Chinese government have implemented the two-child policy and the population did not show a significant rise? The author conducted the following analysis.

From the above predictions, although China implemented a Two-child policy. However, the population did not explode and the population grew steadily. For the "80s" and "90s", after they entered the society, China's housing prices have been too high to afford. The pressure of life is quite large. And there is a certain pressure to raise the second child. Chinese government thought comprehensive second child will gradually improve China's family structure and have the opportunity to return to a normal social state. However, it is still very difficult to reverse China's aging trend. Through the analysis of the situation, people's fertility desire is not high at present, and population change and relatively stable growth in previous years are inevitable phenomena. The prediction results of the model are generally consistent with the actual results. It proves the accuracy of the model once again.

The main reasons for this are the increase in the cost of raising the child, the cost of education, the overwhelming pressure on the elderly, and the lack of housing. The children under the one-child policy of the year are now just couples of the right age. Therefore, if the Chinese government hopes to increase the population and ease the aging of the population, a single second-child policy is not enough, and it also needs supportive policies such as education, house prices, food security, and pension security policies to fundamentally solve the problem. So, in the implementation of the two-child policy, relevant supporting policies must be implemented to enable the second child to achieve the desired results.

\section{References}

Album: China cancels one-child policy. From http://www.bbc.com/zhongwen/simp/indepth/cluster_china_abandon_one_child_policy

Jingmin Wang, Yanfu Sun, \& Junjie Kang. (2010). Evaluation of Electric Power Enterprises' Competitiveness Based on Entropy Method and Improved TOPSIS Method [J]. Journal of North China Electric Power University, 37(6), 61-4.

Kuhn, M. \& Johnson, K. (2017). Applied Predictive Modeling(1rd ed). World Publishing Corporation(Chapter 2),. 6.

National data (2018). In National Bureau of Statistics of the People's Republic of China from http://www.stats.gov.cn/

Two-child policy. From https://en.m.wikipedia.org/wiki/Two-child_policy/

Yang Liu, Yazhe Chen, Yangsong Li, et al. (2009). Product Generalized Quality Comprehensive Evaluation Method Based on Analytic Hierarchy Process and Entropy Method [J]. Chinese Journal of Construction Machinery, 4.

Yi Zhang, Yong Wei, \& Changwei Xiong. (2007). A New Optimization Method for Grey Model GM (1, 1) [J]. System Engineering Theory and Practice, 27(4), 141-6. 


\section{Copyrights}

Copyright for this article is retained by the author(s), with first publication rights granted to the journal.

This is an open-access article distributed under the terms and conditions of the Creative Commons Attribution license (http://creativecommons.org/licenses/by/4.0/). 